Bulletin of the Section of Logic

Volume 47/4 (2018), pp. 265-281

http://dx.doi.org/10.18778/0138-0680.47.4.03

Andrzej Indrzejczak

\title{
RULE-GENERATION THEOREM AND ITS APPLICATIONS
}

\begin{abstract}
In several applications of sequent calculi going beyond pure logic, an introduction of suitably defined rules seems to be more profitable than addition of extra axiomatic sequents. A program of formalization of mathematical theories via rules of special sort was developed successfully by Negri and von Plato. In this paper a general theorem on possible ways of transforming axiomatic sequents into rules in sequent calculi is proved. We discuss its possible applications and provide some case studies for illustration.
\end{abstract}

Keywords: sequent calculus, cut elimination, proof theory, extralogical rules.

\section{Introduction}

We consider the problem of extending sequent calculus (SC) with arbitrary number of rules in a way which allows for preservation of good prooftheoretical properties, in particular, cut elimination. This is a vital question for many, apparently diverse, areas like formalization of mathematical theories, building uniform SC for families of logics, expressing hypothetical reasoning or some other specific discourses which may be expressed by means of collections of atomic sequents (Horn clauses, Post rules) in the setting of SC. The problems mentioned above were often investigated rather separately but it seems that they can be treated in a unified way described below.

In the most systematic way the problem was considered in the context of formal mathematical theories. Negri and von Plato [26] described four 
approaches to the question of how (axiomatic) formal theory $\mathrm{T}$ can be expressed in the setting of SC:

1. Addition of axiomatic sequents of the form $\Rightarrow \varphi$ for each axiom $\varphi$ of $\mathrm{T}$.

2. Addition of "mathematical basic sequents" which consist of atomic formulae obtained from axioms by some kind of decomposition eliminating logical constants.

3. Addition of axioms of $\mathrm{T}$ as a context in the antecedents of all provable sequents.

4. Addition of new rules corresponding to axioms of $\mathrm{T}$.

All these approaches were applied in proof theoretic works on mathematical theories which use SC. For example, the second and the third approach were already applied by Gentzen $[9,10]$, the last one was applied first by Curry [7]. We may apply them also if we want to extend SC adequate for some basic logic to obtain its strengtenings. For example let us consider some $\mathrm{SC}$ for modal logic $\mathbf{K}$ and its extensions to some stronger (normal) modal logic like $\mathbf{T}$ or $\mathbf{S} 4$. In fact, approaches $1-3$ are rarely applied and the standard solution is to provide suitable rules ${ }^{1}$. In some cases it is simply an addition of new rules to SC for $\mathbf{K}$ (standard SC formalizations of $\mathbf{T}$ provide good example) but very often it is just a modification of the original rules (like for $\mathbf{S 4}$ ). Unfortunately, no general unified method is available, at least in the case of standard SC, and most solutions are made ad hoc, sometimes with bad impact on proof theoretical properties. For example, there is no constructive proof of cut elimination for standard SC for S5 and only indirect ways of providing such results are known (see e.g. [15]). In case of other families of non-classical logics the situation is similar, only for the family of a few substructural logics it looks better since we obtain strengtenings in a modular way by means of addition/modification of some structural rules ${ }^{2}$. A lot of work was done in the framework of generalised SC, like display or hypersequent calculi, to provide procedures for automatic generation of rules from axioms in different families of non-

\footnotetext{
${ }^{1}$ See for example surveys in Fitting [8], Wansing [34, 35], Indrzejczak [11] or Poggiolesi [30].

${ }^{2}$ But even here one may notice several irregularities in case of relevance and manyvalued logics, see in particular, Chapter 4 of Paoli [29] and Chapter 5-6 of Bimbo [1].
} 
classical $\operatorname{logics}^{3}$. In fact, some of the provided solutions were devised also for standard SC but with rule constraints, like in Lellmann and Pattinson [22].

Formal representation of hypothetical reasoning was investigated by Schroeder-Heister [31]. He provided a general account of the problem and distinguished three main approaches:

1. Placeholder view in two variants:

(a) elimination by discharge of hypotheses;

(b) elimination by substitution of proofs of hypotheses.

2. No-assumption view.

3. Bidirectional view.

The lack of space makes a detailed presentation rather difficult hence we direct the reader to [31] and only briefly point out the most important things from the standpoint of this work. These approaches, perhaps with the exception of the last, are not necessarily dependent on the specific kind of formal system although the first (in both versions) is considered in the setting of natural deduction (ND) and the second most naturally arise from the consideration of Hilbert systems. Apparently, only the last approach is based on the specific features of SC. However, if we put all of them in the setting of SC we may observe that this issue shows close relationship to the problem of handling theories in the framework of SC and expressing definitions by means of rules. Again it is connected with the possibility of decomposition of complex assumptions into their (atomic) parts, finally formalised either as sequents or as different kinds of rules.

Certainly, also other areas may be found where it is vital to apply SC beyond logic in a satisfactory way. But which kind of application may be claimed to be satisfactory? From the four approaches listed by Negri and von Plato the first two do not admit full elimination of cut. The third one allows for cut elimination but, similarly as the first approach, does not allow for proof theoretic analysis of added machinery of axioms. The last approach provides the most attractive solution. As we mentioned, it was used in the formalization of non-classical logics from the very beginning of the application of SC in this field (see e.g. Ohnishi and Matsumoto $[28])$. This area of investigation also shows that not all types of rules

\footnotetext{
${ }^{3}$ Perhaps the earliest work in this field is Kracht [18], more recent ones include Ciabattoni, Galatos, Terui [4], Ciabattoni and Ramanayake [6] or Lellmann [21, 20].
} 
are well-behaved and that in many cases one must look for some more general solutions, especially if cut elimination is wanted. In case of the formalization of mathematical theories Curry [7] initiated the approach based on the application of rules expressing axioms but a systematic study of the rule-based approach was developed much later by Negri and von Plato $[26,27]$ who demonstrated its usefulness. They have shown that cut elimination, generalised subformula property, and other important results hold for SC enriched with particular kind of nonlogical rules. One specific strategy was developed in [26] and enriched with more examples and other complementary strategies in [27].

In what follows we consider the problem in full generality and ask how many equivalent rules of different shape may be defined for arbitrary sequent. Some partial result of this sort was mentioned in Indrzejczak [11] whereas the general result was presented in Indrzejczak [12]. We restrict considerations to classical logic since it is most often used as a basis for extensions. A general result concerning generation of rules from sequents provided below describes in a combinatorial way the space of possible choices. On the basis of this list we can, in case of any specific task, find such a set of rules which is the best for our purposes. Clearly, the influence of the chosen options on preservation of good proof-theoretical properties like cutelimination, subformula-property or proof-search strategies is particularly important.

In section 2 we prove a lemma establishing equivalence between three simple forms of sequents and the corresponding rules. Some examples are provided to illustrate its usefulness. Despite the practical sufficiency of this result we will formulate and prove a generalised version of this lemma providing equivalent rules for any finite sequent in section 3. Eventually we discuss some possible applications, extensions, and strategies for proving cut elimination with the particular form of rules in the last section.

\section{Useful Cases}

Let us consider some standard SC for classical logic with primitive cut rule like Gentzen's LK but with sequents built from multisets to avoid inessential complications. We also prefer to present all two-premiss rules in the multiplicative (or with independent contexts) version but essentially it is Gentzen's LK. The calculus consists of the following structural and logical rules: 


$$
\begin{aligned}
& (A X) \varphi \Rightarrow \varphi \\
& \text { (Cut) } \frac{\Gamma \Rightarrow \Delta, \varphi \quad \varphi, \Pi \Rightarrow \Sigma}{\Gamma, \Pi \Rightarrow \Delta, \Sigma} \\
& (W \Rightarrow) \frac{\Gamma \Rightarrow \Delta}{\varphi, \Gamma \Rightarrow \Delta} \\
& (\Rightarrow W) \frac{\Gamma \Rightarrow \Delta}{\Gamma \Rightarrow \Delta, \varphi} \\
& (C \Rightarrow) \frac{\varphi, \varphi, \Gamma \Rightarrow \Delta}{\varphi, \Gamma \Rightarrow \Delta} \\
& (\Rightarrow C) \frac{\Gamma \Rightarrow \Delta, \varphi, \varphi}{\Gamma \Rightarrow \Delta, \varphi} \\
& (\neg \Rightarrow) \frac{\Gamma \Rightarrow \Delta, \varphi}{\neg \varphi, \Gamma \Rightarrow \Delta} \\
& (\Rightarrow \neg) \frac{\varphi, \Gamma \Rightarrow \Delta}{\Gamma \Rightarrow \Delta, \neg \varphi} \\
& (\wedge \Rightarrow) \frac{\varphi, \psi, \Gamma \Rightarrow \Delta}{\varphi \wedge \psi, \Gamma \Rightarrow \Delta} \\
& (\Rightarrow \wedge) \frac{\Gamma \Rightarrow \Delta, \varphi \quad \Pi \Rightarrow \Sigma, \psi}{\Gamma, \Pi \Rightarrow \Delta, \Sigma, \varphi \wedge \psi} \\
& (\vee \Rightarrow) \frac{\varphi, \Gamma \Rightarrow \Delta \quad \psi, \Pi \Rightarrow \Sigma}{\varphi \vee \psi, \Gamma, \Pi \Rightarrow \Delta, \Sigma} \\
& (\Rightarrow \vee) \quad \frac{\Gamma \Rightarrow \Delta, \varphi, \psi}{\Gamma \Rightarrow \Delta, \varphi \vee \psi} \\
& (\rightarrow \Rightarrow) \frac{\Gamma \Rightarrow \Delta, \varphi \quad \psi, \Pi \Rightarrow \Sigma}{\varphi \rightarrow \psi, \Gamma, \Pi \Rightarrow \Delta, \Sigma} \\
& (\Rightarrow \rightarrow) \frac{\varphi, \Gamma \Rightarrow \Delta, \psi}{\Gamma \Rightarrow \Delta, \varphi \rightarrow \psi} \\
& (\forall \Rightarrow) \frac{\varphi[x / t], \Gamma \Rightarrow \Delta}{\forall x \varphi, \Gamma \Rightarrow \Delta} \\
& (\Rightarrow \forall)^{1} \frac{\Gamma \Rightarrow \Delta, \varphi[x / a]}{\Gamma \Rightarrow \Delta, \forall x \varphi} \\
& (\exists \Rightarrow)^{1} \frac{\varphi[x / a], \Gamma \Rightarrow \Delta}{\exists x \varphi, \Gamma \Rightarrow \Delta} \\
& (\Rightarrow \exists) \frac{\Gamma \Rightarrow \Delta, \varphi[x / t]}{\Gamma \Rightarrow \Delta, \exists x \varphi}
\end{aligned}
$$

1. where $a$ is not in $\Gamma, \Delta, \varphi$.

How can we change arbitrary sequents added to LK into rules having the same power of proving new sequents? We start with a lemma of rather concrete character, concerned with generation of rules equivalent to sequents of the form $\varphi \Rightarrow \psi, \varphi, \psi \Rightarrow \chi$ and $\varphi \Rightarrow \psi, \chi$. Taking into account that quite often we deal with sequents or axioms (with $\rightarrow$ instead of $\Rightarrow$ and $\wedge$ or $\vee$ added) of this form this lemma is practically sufficient for most cases. It allows for fast establishing of equivalency of different SC formalizations of some logics/theories, and for quick generation of new variants having desirable properties. For unification of results and simplification of proofs we do not mention applications of structural rules, and formulate all rules with many premisses in multiplicative version, but they may be proved also for additive versions without any difficulties.

LEMma 1. The following schemata of sequents and rules collected in three groups are interderivable in $L K$ : 
A: for (1) $\varphi \Rightarrow \psi$ :
(2) $\frac{\psi, \Gamma \Rightarrow \Delta}{\varphi, \Gamma \Rightarrow \Delta}$
(3) $\frac{\Gamma \Rightarrow \Delta, \varphi}{\Gamma \Rightarrow \Delta, \psi}$
(4) $\frac{\Gamma \Rightarrow \Delta, \varphi \quad \psi, \Pi \Rightarrow \Sigma}{\Gamma, \Pi \Rightarrow \Delta, \Sigma}$

B: for (1) $\varphi, \psi \Rightarrow \chi$ :
(2) $\frac{\chi, \Gamma \Rightarrow \Delta}{\varphi, \psi, \Gamma \Rightarrow \Delta}$
(3) $\frac{\Gamma \Rightarrow \Delta, \varphi}{\psi, \Gamma \Rightarrow \Delta, \chi}$
(4) $\frac{\Gamma \Rightarrow \Delta, \psi}{\varphi, \Gamma \Rightarrow \Delta, \chi}$
(5) $\frac{\Gamma \Rightarrow \Delta, \varphi \quad \Pi \Rightarrow \Sigma, \psi}{\Gamma, \Pi \Rightarrow \Delta, \Sigma, \chi}$
(6) $\frac{\Gamma \Rightarrow \Delta, \varphi \quad \chi, \Pi \Rightarrow \Sigma}{\psi, \Gamma, \Pi \Rightarrow \Delta, \Sigma}$
(7) $\frac{\Gamma \Rightarrow \Delta, \psi \quad \chi, \Pi \Rightarrow \Sigma}{\varphi, \Gamma, \Pi \Rightarrow \Delta, \Sigma}$
(8) $\frac{\Gamma \Rightarrow \Delta, \varphi \quad \Pi \Rightarrow \Sigma, \psi \quad \chi, \Lambda \Rightarrow \Theta}{\Gamma, \Pi, \Lambda \Rightarrow \Delta, \Sigma, \Theta}$

C: for (1) $\varphi \Rightarrow \psi, \chi$ :
(2) $\frac{\Gamma \Rightarrow \Delta, \varphi}{\Gamma \Rightarrow \Delta, \psi, \chi}$
(3) $\frac{\psi, \Gamma \Rightarrow \Delta}{\varphi, \Gamma \Rightarrow \Delta, \chi}$
(4) $\frac{\chi, \Gamma \Rightarrow \Delta}{\varphi, \Gamma \Rightarrow \Delta, \psi}$
(5) $\frac{\psi, \Gamma \Rightarrow \Delta \quad \chi, \Pi \Rightarrow \Sigma}{\varphi, \Gamma, \Pi \Rightarrow \Delta, \Sigma}$
(6) $\frac{\Gamma \Rightarrow \Delta, \varphi \quad \chi, \Pi \Rightarrow \Sigma}{\Gamma, \Pi \Rightarrow \Delta, \Sigma, \psi}$
(7) $\frac{\Gamma \Rightarrow \Delta, \varphi \quad \psi, \Pi \Rightarrow \Sigma}{\Gamma, \Pi \Rightarrow \Delta, \Sigma, \chi}$
(8) $\frac{\Gamma \Rightarrow \Delta, \varphi \quad \psi, \Pi \Rightarrow \Sigma \quad \chi, \Lambda \Rightarrow \Theta}{\Gamma, \Pi, \Lambda \Rightarrow \Delta, \Sigma, \Theta}$

Proof: We prove it for A and B. For A.

1 . $\Longrightarrow 2 .:$ It is suficient to apply (Cut) to 1 and to the premiss in 2 .

2 . $\Longrightarrow 3$. From axiom $\psi \Rightarrow \psi$ by 2 we obtain $\varphi \Rightarrow \psi$ which by (Cut) with premiss in 3 yields the conclusion.

$3 . \Longrightarrow 4 .:$ the following schema schows derivability:

$$
\frac{\Gamma \Rightarrow \Delta, \varphi \quad \text { (3.) } \frac{\varphi \Rightarrow \varphi}{\varphi \Rightarrow \psi} \quad \psi, \Gamma^{\prime} \Rightarrow \Delta^{\prime}}{\varphi, \Gamma^{\prime} \Rightarrow \Delta^{\prime}}(C u t)
$$

4. $\Longrightarrow 1 .:$ From $\varphi \Rightarrow \varphi$ and $\psi \Rightarrow \psi$ by 4 we get 1 .

For B.

1. $\Longrightarrow 2 .:$ It is enough to apply (Cut) to 1 and to premiss 2 to obtain the conclusion.

2. $\Longrightarrow 3 .:$ From $\chi \Rightarrow \chi$ by 2 we get $\varphi, \psi \Rightarrow \chi$ which by (Cut) with premiss 3 yields the conclusion. 
3. $\Longrightarrow 4$.: analogous, with 3 applied to $\varphi \Rightarrow \varphi$.

4. $\Longrightarrow 5$.: analogous, but 4 is applied not to axiom but to premiss 5 of the shape $\Gamma^{\prime} \Rightarrow \Delta^{\prime}, \psi$ in order to get the conclusion by (Cut) on the second premiss.

$5 . \Longrightarrow 6 .:$ the following schema schows derivability:

$$
\text { (5.) } \frac{\Gamma \Rightarrow \Delta, \varphi \quad \psi \Rightarrow \psi}{\frac{\psi, \Gamma \Rightarrow \Delta, \chi}{\psi, \Gamma, \Gamma^{\prime} \Rightarrow \Delta, \Delta^{\prime}} \quad \chi, \Gamma^{\prime} \Rightarrow \Delta^{\prime}}(C u t)
$$

6. $\Longrightarrow 7 .:$ the following schema schows derivability:

$$
\frac{\Gamma \Rightarrow \Delta, \psi \quad \frac{\varphi \Rightarrow \varphi \quad \chi, \Gamma^{\prime} \Rightarrow \Delta^{\prime}}{\psi, \varphi, \Gamma^{\prime} \Rightarrow \Delta^{\prime}}(C u t)}{\varphi, \Gamma, \Gamma^{\prime} \Rightarrow \Delta, \Delta^{\prime}}
$$

7. $\Longrightarrow$ 8.: the following schema schows derivability:

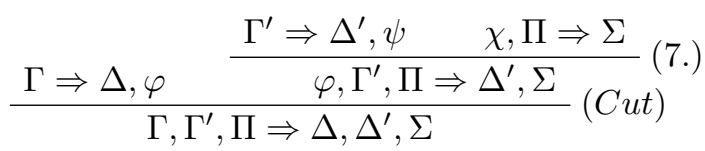

8. $\Longrightarrow 1$.: From $\varphi \Rightarrow \varphi, \psi \Rightarrow \psi$ and $\chi \Rightarrow \chi$ by 8 we deduce 1 .

In principle, we are interested in the application of these results to formalization of extralogical contexts. However, their character is general enough to cover also logical applications. Let us pause for a moment to provide some examples of the possible applications of this lemma.

We start with the case of formalizing $\vee$. One can easily recognize that the rule $(\Rightarrow \vee)$ for (additive) disjunction is captured by schema A.3. Hence, by the lemma, we can use instead either the sequent $\varphi \Rightarrow \varphi \vee \psi$ or the following rules:

$$
(2 \vee) \frac{\varphi \vee \psi, \Gamma \Rightarrow \Delta}{\varphi, \Gamma \Rightarrow \Delta} \quad(4 \vee) \frac{\Gamma \Rightarrow \Delta, \varphi \quad \varphi \vee \psi, \Pi \Rightarrow \Sigma}{\Gamma, \Pi \Rightarrow \Delta, \Sigma}
$$

On the other hand, $(\vee \Rightarrow)$ is captured by the schema C.5. (with $\varphi:=$ $\psi \vee \chi)$ which is equivalent to sequents of the form $\psi \vee \chi \Rightarrow \psi, \chi$ and generates the following equivalents:
( 22$) \frac{\Gamma \Rightarrow \Delta, \psi \vee \chi}{\Gamma \Rightarrow \Delta, \psi, \chi}$
$(\vee 3) \frac{\psi, \Gamma \Rightarrow \Delta}{\psi \vee \chi, \Gamma \Rightarrow \Delta, \chi}$
$(\vee 4) \frac{\chi, \Gamma \Rightarrow \Delta}{\psi \vee \chi, \Gamma \Rightarrow \Delta, \psi}$
( 6$) \frac{\Gamma \Rightarrow \Delta, \psi \vee \chi \quad \chi, \Pi \Rightarrow \Sigma}{\Gamma, \Pi \Rightarrow \Delta, \Sigma, \psi}$
( 7$) \frac{\Gamma \Rightarrow \Delta, \psi \vee \chi \quad \psi, \Pi \Rightarrow \Sigma}{\Gamma, \Pi \Rightarrow \Delta, \Sigma, \chi}$ 
( V8) $\frac{\Gamma \Rightarrow \Delta, \psi \vee \chi \quad \psi, \Pi \Rightarrow \Sigma \quad \chi, \Lambda \Rightarrow \Theta}{\Gamma, \Pi, \Lambda \Rightarrow \Delta, \Sigma, \Theta}$

A good illustration of the case B is provided by Substitution Principle which is often used to formalize identity relation (in addition to reflexivity law). In the framework of SC it is of the form $t_{1}=t_{2}, \varphi\left[x / t_{2}\right] \Rightarrow \varphi\left[x / t_{1}\right]$ and was used e.g. by Troelstra and Schwichtenberg [33], or in a slightly modified way by Takeuti [32] and Buss [3]. By the lemma we can generate the following equivalents:

$$
\begin{aligned}
& (2=) \frac{\varphi\left[x / t_{2}\right], \Gamma \Rightarrow \Delta}{t_{1}=t_{2}, \varphi\left[x / t_{1}\right], \Gamma \Rightarrow \Delta} \quad(3=) \frac{\Gamma \Rightarrow \Delta, \varphi\left[x / t_{1}\right]}{t_{1}=t_{2}, \Gamma \Rightarrow \Delta, \varphi\left[x / t_{2}\right]} \\
& (4=) \frac{\Gamma \Rightarrow \Delta, t_{1}=t_{2}}{\varphi\left[x / t_{1}\right], \Gamma \Rightarrow \Delta, \varphi\left[x / t_{2}\right]} \quad(5=) \frac{\Gamma \Rightarrow \Delta, t_{1}=t_{2} \quad \Pi \Rightarrow \Sigma, \varphi\left[x / t_{1}\right]}{\Gamma, \Pi \Rightarrow \Delta, \Sigma, \varphi\left[x / t_{2}\right]} \\
& (6=) \frac{\Gamma \Rightarrow \Delta, t_{1}=t_{2} \quad \varphi\left[x / t_{2}\right], \Pi \Rightarrow \Sigma}{\varphi\left[x / t_{1}\right], \Gamma, \Pi \Rightarrow \Delta, \Sigma} \\
& (7=) \frac{\Gamma \Rightarrow \Delta, \varphi\left[x / t_{1}\right] \quad \varphi\left[x / t_{2}\right], \Pi \Rightarrow \Sigma}{t_{1}=t_{2}, \Gamma, \Pi \Rightarrow \Delta, \Sigma} \\
& (8=) \frac{\Gamma \Rightarrow \Delta, t_{1}=t_{2} \quad \Pi \Rightarrow \Sigma, \varphi\left[x / t_{1}\right] \quad \varphi\left[x / t_{2}\right], \Lambda \Rightarrow \Theta}{\Gamma, \Pi, \Lambda \Rightarrow \Delta, \Sigma, \Theta}
\end{aligned}
$$

From these variants Negri and von Plato applied $(2=)$ (but with the repetition of active formulae in the premiss to save admissibility of contraction) and $(4=)$ in [27]), Manzano [23] used $(3=)$, Nagashima [25] used $(7=)$; to mention just a few examples. Another example of the application of schema B is provided by the rule of elimination of $\forall$ in free logics. Let $E$ denote the existence predicate, suitable sequent is of the form: $\forall x \varphi, E t \Rightarrow \varphi[x / t]$. The reader can easily provide 7 equivalent rules. Note that in SC formalizations of free logics either rules of type B2 or of type B7 are used.

\section{Rule-Generation Theorem}

Now the main result. We can generalise Lemma 1 in the following way:

THEOREM 1. For any sequent $\Gamma \Rightarrow \Delta$ with $\Gamma=\left\{\varphi_{1}, \ldots, \varphi_{k}\right\}$ and $\Delta=$ $\left\{\psi_{1}, \ldots, \psi_{n}\right\}, k \geq 0, n \geq 0, k+n \geq 1$ there are $2^{k+n}-1$ equivalent rules captured by the general schema: 


$$
\frac{\Pi_{1}, \Rightarrow \Sigma_{1}, \varphi_{1}, \ldots, \Pi_{i} \Rightarrow \Sigma_{i}, \varphi_{i} \quad \psi_{1}, \Pi_{i+1} \Rightarrow \Sigma_{i+1}, \ldots, \psi_{j}, \Pi_{i+j} \Rightarrow \Sigma_{i+j}}{\Gamma^{-i}, \Pi_{1}, \ldots, \Pi_{i}, \Pi_{i+1}, \ldots, \Pi_{i+j} \Rightarrow \Sigma_{1}, \ldots, \Sigma_{i}, \Sigma_{i+1}, \ldots, \Sigma_{i+j} \Delta^{-j}}
$$
where $\Gamma^{-i}=\Gamma-\left\{\varphi_{1}, \ldots, \varphi_{i}\right\}$ and $\Delta^{-j}=\Delta-\left\{\psi_{1}, \ldots, \psi_{j}\right\}$ for $0 \leq i \leq k, 0 \leq$ $j \leq n$.

A comment on the schema may be helpful before we provide a proof. In general, we define rules taking arbitrary number of formulae from the antecedent or succedent, and for every such formula we create a premisssequent where an item from the antecedent $\left(\varphi_{i}\right)$ is put in the succedent, and the element of the succedent $\left(\psi_{j}\right)$ is put in the antecedent of a premisssequent. The remaining formulae from input sequent are collected into sets $\Gamma^{-i}$ and $\Delta^{-j}$ of the conclusion-sequent. Extreme cases are empty sets $\Gamma^{-k}$ and $\Delta^{-n}$. A rule has $k+n$ premisses, each one for every formula from inputsequent, and conclusion contains only unions of parameters from premisses. On the other hand, if we consider a situation with $\Gamma^{-0}$ and $\Delta^{-0}$, then our schema covers also a case with no premisses at all, i.e. our input sequent.

Proof: A proof goes by induction on $k+n$. For basic cases with $k=$ $1, n=0$ or $k=0, n=1$ (sequents of the form $\varphi \Rightarrow$ and $\Rightarrow \psi$ ) there is only one equivalent rule: $\Gamma \Rightarrow \Delta, \varphi / \Gamma \Rightarrow \Delta$ and $\psi, \Gamma \Rightarrow \Delta / \Gamma \Rightarrow \Delta$, i.e. we have $2^{1}-1$ rules. Also the case of $n=k=1$ (as well as two cases for $k+n=3$ ) was shown above to satisfy the claim.

For the inductive step we assume that for some $S=\varphi_{1}, \ldots, \varphi_{k} \Rightarrow$ $\psi_{1}, \ldots, \psi_{n}$ the claim holds and we will show that it holds if we add some $\chi$ to the antecedent or succedent of $S$. First consider the addition of $\chi$ to the antecedent which we schematize as $\chi, S$. Let $i=2^{k+n}$, so we have $S$ and $k-1$ equivalent rules by assumption. From each rule we generate 2 new rules in the following way: (a) either add $\chi$ to the antecedent of the conclusion or (b) add the additional premiss $\Pi \Rightarrow \Sigma$, $\chi$ with arbitrary $\Pi, \Sigma$. So if the rule equivalent to $S$ has the shape: $S_{1}, \ldots, S_{j} / S_{j+1}$ for $i \geq j \geq 1$, we got either: (a) $S_{1}, \ldots, S_{j} / \chi, S_{j+1}$ or (b) $\Pi \Rightarrow \Sigma, \chi, S_{1}, \ldots, S_{j} / \Pi, S_{j+1}, \Sigma$. In case of $S$ we do the same so we obtain (a) a sequent $\chi, S$ and (b) a rule $\Pi \Rightarrow \Sigma, \chi / S$. In total we obtain $2 i-1$ rules and a sequent $\chi$, $S$, i.e. $2^{k+n+1}-1$ rules.

It is easy to show that the new rules are interderivable with $\chi, S$ in the way illustrated in the proof of the preceeding lemma. First, if we take $\chi, S$, then by $j$ applications of (Cut) to all premisses of (a) we will get $\chi, S_{j+1}$. This is because $j$ elements of $S$ are distributed as active formulae 
in premisses in the following way: all active formulae from antecedents of premisses are in the succedent of $S$ and all from succedents are in the antecedent. After $j$ applications of (Cut) the remaining elements of $S$ (if any, i.e. if $k+n>j$ ) with the union of all parameters from $j$ premisses are in $\chi, S_{j+1}$. This way we demonstrate the provability of (a) on the basis of $\chi, S$.

(b) is derivable from (a) by one application of (Cut) in the following way:

$$
\frac{\Pi \Rightarrow \Sigma, \chi \quad \frac{S_{1}, \ldots, S_{j}}{\chi, S_{j+1}}(\text { (a) }}{\Pi, S_{j+1}, \Sigma}(\text { ut })
$$

Eventually, we derive $\chi, S$ by means of (b) from axiom $\chi \Rightarrow \chi$ and $j$ axioms of the form $\varphi_{l} \Rightarrow \varphi_{l}, \psi_{m} \Rightarrow \psi_{m}$, for $l \leq k, m \leq n$.

If we add $\chi$ to the succedent, i.e. we consider a sequent $S, \chi$, the procedure is symmetric: (a) either addition of $\chi$ to the succedent of the conclusion or (b) addition of the extra premiss $\chi, \Pi \Rightarrow \Sigma$.

\section{Benefits}

We already mentioned that this theorem just delimits the space of possible choices in building concrete calculi. The actual choices may depend on different factors. We comment on two, possibly the most important, the shape of the rules and the structure of active formulae.

The variety of forms is fine but not all of them have nice proof-theoretic features. In particular, not all solutions yield cut elimination theorem, but this will be explained later. Of course, not only cut elimination matters, other factors may be decisive since a choice of suitable rules depends on the aims of research. For example, if we are interested in the calculus well suited for (root-first) proof search, a branching factor may be important in the sense that rules with smaller number of premisses may be preferable. Also - for the same reason - we may prefer the rules in which the number of active formulae in premisses is smaller than in the conclusion or, if active formulae are of different complexity, we may prefer rules where they are of lower complexity in premisses. But other needs may force other choices.

A general approach due to Negri and von Plato [26] is based on the application of rules where all active formulae are displayed in antecedents 
(rules of type A.2., B.2., C.5.). It works nicely with Dragalin's strategy of proving cut admissibility for invertible logical (i.e. with no primitive structural rules) calculus G3. The disadvantage of this approach is that we must provide numerous preliminary results concerning invertibility of rules, admissibility of contraction e.t.c. (hence G3 is needed). Moreover, such kind of rules may seem not necessarily the most natural. The same authors in [27] are using SC with rules working only on succedents (A.3., B.5., C.2.,), and even with both kind of rules (theory of linear lattices). It is shown there that some other choices may be more natural for proving different kinds of results.

We prefer to work with rules having all active formula in succedents. Such an approach was first developed by Curry [7] who proved also cut admissibility for systems with rules of this kind. This solution seems to be more proof-construction directed and work nicely with any kind of rules (additive, multiplicative). The choice of LK is also natural in this context since there is no necessity for proving preliminary results on admissibility of structural rules which are crucial for proving the Rule-Generation Theorem. Such choice may be also easily mixed with the strategy of proof of cut elimination based on the approach originally provided for hypersequent calculi by Metcalfe, Olivetti and Gabbay [24] and later extensively used in this framework (see, e.g. Ciabattoni, Metcalfe, Montagna [5], Indrzejczak [13], Kurokawa [19]). However, it is applicable also to standard sequent calculi (e.g. Indrzejczak $[14,16,17])$ and allows for elegant proof which helps to avoid many complexities inherent in other methods of cut elimination proof. In fact this general strategy of proof is somewhat similar to Curry's [7] proof of cut admissibility but simpler in some respects and still based rather on local transformations of proof instead of global ones characteristic for Curry's proof. The proof of cut elimination theorem is based on two lemmata which make a reduction first on the right and next on the left premiss of cut.

Lemma 2 (Right reduction). Let $\mathcal{D}_{1} \vdash \Gamma \Rightarrow \Delta, \varphi$ and $\mathcal{D}_{2} \vdash \varphi^{k}, \Pi \Rightarrow \Sigma$ with $d \mathcal{D}_{1}, d \mathcal{D}_{2}<d \varphi$, and $\varphi$ principal in $\Gamma \Rightarrow \Delta, \varphi$, then we can construct a proof $\mathcal{D}$ such that $\mathcal{D} \vdash \Gamma^{k}, \Pi \Rightarrow \Delta^{k}, \Sigma$ and $d \mathcal{D}<d \varphi$. 
Lemma 3 (Left reduction). Let $\mathcal{D}_{1} \vdash \Gamma \Rightarrow \Delta, \varphi^{k}$ and $\mathcal{D}_{2} \vdash \varphi, \Pi \Rightarrow \Sigma$ with $d \mathcal{D}_{1}, d \mathcal{D}_{2}<d \varphi$, then we can construct a proof $\mathcal{D}$ such that $\mathcal{D} \vdash \Gamma, \Pi^{k} \Rightarrow$ $\Delta, \Sigma^{k}$ and $d \mathcal{D}<d \varphi$.

In both lemmata $d \varphi$ denotes a degree (complexity) of $\varphi$ and $d \mathcal{D}_{i}$ denotes a cut-degree of a derivation $\mathcal{D}_{i}$ which is a maximal degree of cut formula in this derivation. So the proof is based on the strategy of elimination of the maximal cuts not the highest ones like in Gentzen's original strategy. Cut elimination follows easily from the Left Reduction Lemma. Since all additional rules have active formulae on the right only, then the Right Reduction Lemma goes without any changes. All we need is the additional work for the Left Reduction Lemma. But here the problem of the structure of active formulae is vital. Although it is neither necessary nor sufficient for cut elimination, it is important to have rules which consists only of atomic active formulae. Why is this important? Notice that in proofs of cut elimination usually one of the inductive parameters is the complexity of cut formula. If both occurrences of the (complex) cut formulae were introduced immediately above the application of cut on them, we would have to be able to show that this part of the proof may be replaced with series of cuts made on subformulae of this complex cut formula since the latter are eliminable by the induction hypothesis. It is the beauty and strength of the standard rules for logical constants that they are reductive in this sense. However, if some additional rule also generates a complex formula in the conclusion, we must consider also a situation when one complex cut formula was deduced by this nonlogical rule, and the other by suitable logical rule. In such cases a reduction may be not possible ${ }^{4}$. Clearly, in the case of propositional level it is not problematic. In classical logic every formula may be transformed into equivalent conjunctive normal form and each conjunct may be transformed into a basic sequent. Even in intuitionistic logic, despite the failure of normal form theorem, we can obtain suitable reductions, as is shown in Negri and von Plato [26].

With quantified statements we can proceed generally by transforming them into prefix normal form where matrix is again in conjunctive normal form. However, the elimination of quantifiers is generally connected with skolemization and losing equivalence between input formulae and output

\footnotetext{
${ }^{4}$ Indrzejczak [17] presents SC for modal logic with definite descriptions where such problems lead to suitable changes of rules involving identity.
} 
basic sequents. Since preservation of satisfiability is for many cases sufficient it is not a big problem. Note also that there is a variety of classes of quantified formulae which are especially suitable for generation of rules. We illustrate the point with two examples.

One may easily obtain rules for the class of universal implications of the form $\forall x_{1} \ldots x_{k}\left(\varphi_{1} \wedge \cdots \wedge \varphi_{n} \rightarrow \psi_{1} \vee \cdots \vee \psi_{m}\right)$, where all $\varphi$ 's and $\psi$ 's are atomic formulae. For each such universal implication, the general schema of SC rule in our favourite form will be:

$$
\frac{\Gamma \Rightarrow \Delta, \varphi_{1} \quad \ldots \quad \Gamma \Rightarrow \Delta, \varphi_{n}}{\Gamma \Rightarrow \Delta, \psi_{1}, \ldots, \psi_{m}}
$$

It may be strenghtened to the class of basic geometric formulae of the form:

$$
\forall x_{1} \ldots x_{k}\left(\varphi_{1} \wedge \cdots \wedge \varphi_{n} \rightarrow \exists y_{1} \ldots y_{l}\left(\psi_{1} \vee \cdots \vee \psi_{m}\right)\right)
$$

where $k \geqslant 1, l, n, m \geqslant 0, \varphi$ 's are atomic formulae and $\psi$ 's are either atomic formulae or finite conjunctions of atoms.

It seems that this class may be better treated by other approaches. For example, in Braüner [2], for every such basic geometric formula there corresponds a rule of the following form:

$$
\frac{\Gamma \Rightarrow \Delta, \varphi_{1} \ldots \Gamma \Rightarrow \Delta, \varphi_{n} \quad \Psi_{1}, \Gamma \Rightarrow \Delta \ldots \Psi_{m}, \Gamma \Rightarrow \Delta}{\Gamma \Rightarrow \Delta}
$$

where no variables of $y_{1}, \ldots, y_{l}$ occur in $\Gamma, \Delta, \varphi_{1}, \ldots, \varphi_{n}$, and for each $i=1, \ldots m: \Psi_{i}$ is a set of atoms that form conjunction $\psi_{i}$.

Also in case of Negri and von Plato approach we can provide suitable rules of the form:

$$
\frac{\varphi_{1} \ldots \varphi_{n}, \Psi_{1}, \Gamma \Rightarrow \Delta \quad \ldots \quad \varphi_{1} \ldots \varphi_{n}, \Psi_{m}, \Gamma \Rightarrow \Delta}{\varphi_{1} \ldots \varphi_{n}, \Gamma \Rightarrow \Delta}
$$

where no variables of $y_{1}, \ldots, y_{l}$ occur in $\Gamma, \Delta, \varphi_{1}, \ldots, \varphi_{n}$, and for each $i=1, \ldots m: \Psi_{i}$ is a set of atoms that form conjunction $\psi_{i}$.

For our preferred format of rules it is harder, but still possible, to express geometric formulae. Obviously, we can use rules of the form: 


$$
\frac{\Gamma \Rightarrow \Delta, \varphi_{1} \quad \ldots \quad \Gamma \Rightarrow \Delta, \varphi_{n}}{\Gamma \Rightarrow \Delta, \psi_{1}, \ldots, \psi_{m}}
$$

but this time $\psi$-s may be not atomic and the Right Reduction Lemma may fail. Fortunatelly, we may transform every Braüner's rule into finite set of rules of the form:

$$
\frac{\Gamma \Rightarrow \Delta, \varphi_{1} \quad \ldots \quad \Gamma \Rightarrow \Delta, \varphi_{n}}{\Gamma \Rightarrow \Delta, \chi_{1}, \ldots, \chi_{m}}
$$

where for each $i \leq m, \chi_{i}$ is a selected (atomic) element of $\Psi_{i}$, for every combination of these atoms. Thus the number of such rules is $\prod_{i=1}^{m}\left|\Psi_{i}\right|$, where $\left|\Psi_{i}\right|$ is the number of atoms in $\Psi_{i}$. For example, a Braüner's style rule:

$$
\frac{\Gamma \Rightarrow \Delta, \varphi \quad \psi_{1}, \psi_{2}, \Gamma \Rightarrow \Delta \quad \psi_{3}, \psi_{4}, \Gamma \Rightarrow \Delta}{\Gamma \Rightarrow \Delta}
$$

is equivalent to four rules of the form:

$$
\frac{\Gamma \Rightarrow \Delta, \varphi}{\Gamma \Rightarrow \Delta, \psi_{i}, \psi_{k}}
$$

where $i=1$ or $i=2$ and $k=3$ or $k=4$.

It is easy to show that each of these 4 rules is derivable by Braüner's rule:

$$
\begin{aligned}
& (W) \frac{\Gamma \Rightarrow \Delta, \varphi}{\Gamma \Rightarrow \Delta, \psi_{1}, \psi_{3}, \varphi} \quad \frac{\psi_{1} \Rightarrow \psi_{1}}{\psi_{1}, \psi_{2}, \Gamma \Rightarrow \Delta, \psi_{1}, \psi_{3}} \quad \frac{\psi_{3} \Rightarrow \psi_{3}}{\psi_{3}, \psi_{4}, \Gamma \Rightarrow \Delta, \psi_{1}, \psi_{3}} \\
& \Gamma \Rightarrow \Delta, \psi_{1}, \psi_{3}
\end{aligned}
$$

The other direction is harder but conceptually easy. We can derive:

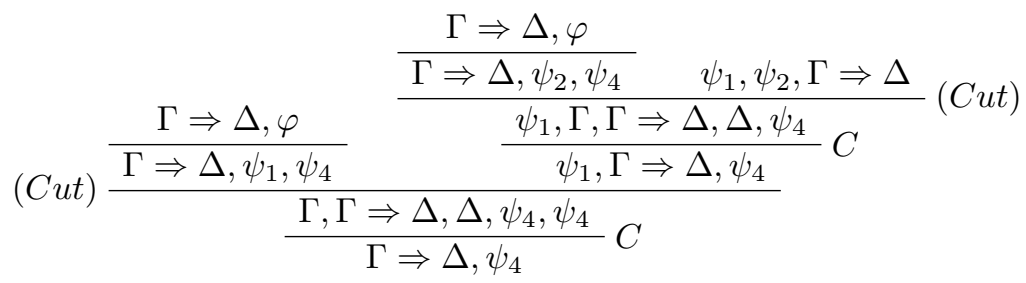


and

$$
(C u t) \frac{\frac{\Gamma \Rightarrow \Delta, \varphi}{\Gamma \Rightarrow \Delta, \psi_{2}, \psi_{3}}}{\left(C \frac{\Gamma, \Gamma \Rightarrow \Delta, \Delta, \psi_{3}, \psi_{3}}{\psi_{2}, \Gamma \Rightarrow \Delta, \psi_{3}}\right.}{ }^{\frac{\Gamma, \psi_{1}, \psi_{3}}{\Gamma \Rightarrow \Delta, \psi_{3}}}(C u t)
$$

By cut and contraction we obtain $\Gamma \Rightarrow \Delta$. One may easily notice that all four rules are necessary to prove derivability of Braüner's rule. In a similar way we can provide proofs of equivalence for any $k, n$ and for conjunctions of any length. In this way we can also cover any such theory over LK (or any other SC for classical logic) with our general cut elimination theorem.

\section{Acknowledgements}

The results reported in this paper are supported by the National Science Centre, Poland (grant number: DEC-2017/25/B/HS1/01268).

\section{References}

[1] K. Bimbo, Proof Theory, CRC Press 2015.

[2] T. Braüner, Hybrid Logic and its Proof-Theory, Roskilde 2009.

[3] S. R. Buss, An Introduction to Proof Theory [in:] S. Buss (ed.) Handbook of Proof Theory, Elsevier 1998.

[4] A. Ciabattoni, N. Galatos and K. Terui, From axioms to analytic rules in nonclassical logics, [in:] LICS (2002), pp. 229-240, IEEE Computer Society, 2008.

[5] A. Ciabattoni, G. Metcalfe and F. Montagna, Algebraic and proof-theoretic characterizations of truth stressers for MTL and its extensions, Fuzzy Sets and Systems 161(3) (2010), pp. 369-389.

[6] A. Ciabattoni and R. Ramanayake, Structural extensions of display calculi: a general recipe, WoLLIC 2013, LNCS pp. 81-95, Springer 2013. 
[7] H. B. Curry, Foundations of Mathematical Logic, McGraw-Hill, New York 1963.

[8] M. Fitting, Proof Methods for Modal and Intuitionistic Logics, Reidel, Dordrecht 1983.

[9] G. Gentzen, Untersuchungen über das Logische Schliessen, Mathematische Zeitschrift 39 (1934), pp. 176-210 and pp. 405-431.

[10] G. Gentzen, Neue Fassung des Widerspruchsfreiheitsbeweises für die reine Zahlentheorie, Forschungen zur Logik und zur Grundlegung der exakten Wissenschaften, New Series 4, Leipzig, pp. 19-44, 1938.

[11] A. Indrzejczak, Natural Deduction, Hybrid Systems and Modal Logics, Springer 2010.

[12] A. Indrzejczak, Sequent Calculi in Classical Logic [in polish], Lodz University Publications 2013.

[13] A. Indrzejczak, Eliminability of Cut in Hypersequent Calculi for some Modal Logics of Linear Frames, Information Processing Letters 115/2 (2015), pp. $75-81$.

[14] A. Indrzejczak, Simple Cut Elimination Proof for Hybrid Logic, Logic and Logical Philosophy 25/2 (2016), pp. 129-141.

[15] A. Indrzejczak, Simple Decision Procedure for S5 in Standard Cut-Free Sequent Calculus, Bulletin of the Section of Logic 45:1 (2016), pp. 95-102.

[16] A. Indrzejczak, Fregean Description Theory in Proof-Theoretical Setting, Logic and Logical Philosophy, Vol. 28, No 1 (2019), pp. 137-155.

[17] A. Indrzejczak, Cut-Free Modal Theory of Definite Descriptions, [in:] G. Bezhanishvili et al. (eds.) Advances in Modal Logic 12, pp. 387-406, College Publications 2018.

[18] M. Kracht, Power and weakness of the modal display calculus, [in:] H. Wansing (ed.) Proof Theory of Modal Logic, pp. 93-121, Kluwer 1996.

[19] H. Kurokawa, Hypersequent Calculi for Modal Logics Extending S4, [in:] New Frontiers in Artificial Intelligence, pp. 51-68, Springer 2014.

[20] B. Lellmann, Axioms vs hypersequent rules with context restrictions, [in:] Proceedings of IJCAR, pp. 307-321, Springer 2014.

[21] B. Lellmann, Hypersequent rules with restricted contexts for propositional modal logics, Theoretical Computer Science.

[22] B. Lellmann and D. Pattinson, Correspondence between modal Hilbert axioms and sequent rules with an application to S5, [in:] TABLEAUX 2013, pp. 219-233, Springer 2013.

[23] M. Manzano, Model Theory, Oxford University Press, Oxford 1999. 
[24] G. Metcalfe, N. Olivetti and D. Gabbay, Proof Theory for Fuzzy Logics, Springer 2008.

[25] T. Nagashima, An extension of the Craig-Schütte interpolation theorem, Annals of the Japan Association for the Philosophy of Science 3 (1966), pp. $12-18$.

[26] S. Negri and J. von Plato, Structural Proof Theory, Cambridge University Press, Cambridge 2001.

[27] S. Negri and J. von Plato, Proof Analysis, Cambridge University Press, Cambridge 2011.

[28] M. Ohnishi and K. Matsumoto, Gentzen Method in Modal Calculi I, Osaka Mathematical Journal 9 (1957), pp. 113-130.

[29] F. Paoli, Substructural Logics: A Primer, Kluwer, Dordrecht 2002.

[30] F. Poggiolesi, Gentzen Calculi for Modal Propositional Logic, Springer 2011.

[31] P. Schroeder-Heister, Open Problems in Proof-theoretic Semantics, [in:] T. Piecha, P. Schroeder-Heister (eds.) Advances in Proof-theoretic Semantics, pp. 253-283, Springer 2016.

[32] G. Takeuti, Proof Theory, North-Holland, Amsterdam 1987.

[33] A. S. Troelstra and H. Schwichtenberg, Basic Proof Theory, Oxford University Press, Oxford 1996.

[34] H. Wansing, Displaying Modal Logics, Kluwer Academic Publishers, Dordrecht 1999.

[35] H. Wansing, Sequent Systems for Modal Logics, [in:] D. Gabbay, F. Guenthner (eds.), Handbook of Philosophical Logic, vol. IV, pp. 89-133, Reidel Publishing Company, Dordrecht 2002.

Department of Logic

University of Łódź

Lindleya $3 / 5$

90-131 Łódź

e-mail: andrzej.indrzejczak@filozof.uni.lodz.pl 